\title{
Comparison of IP, MPLS and MPLS RSVP-TE Networks using OPNET
}

\author{
O. Akinsipe \\ School of Engineering and Design, \\ Brunel University, London, United \\ Kingdom
}

\author{
F. Goodarzi \\ School of Engineering and Design, \\ Brunel University, London, United \\ Kingdom
}

\author{
M. Li \\ School of Engineering and Design, \\ Brunel University, London, United \\ Kingdom
}

\begin{abstract}
The main components of a network are vital to its enhancements by providing reliable services. Different technologies have been put in place to enhance application services in a network. MPLS is one of such technologies and it provides a reliable delivery of application services. It delivers services with low delays, low losses and high speed of transmission. Due to the Traffic Engineering feature of MPLS, it can be used to efficiently utilize network resources as well as implement real-time applications (voice and video). Signalling protocols such as RSVP-TE and CR-LDP are used for Traffic Engineering in MPLS.

In this research, the modelling of IP, MPLS and MPLS RSVP-TE (with path reserved for voice traffic) networks are presented and the performance parameters of the networks are compared. OPNET modeler 16.0 is used to simulate all the networks and the comparison is made for parameters such as throughput, utilization and voice jitter.
\end{abstract}

\section{General Terms}

IP, MPLS, Real-time applications, Traffic Engineering

\section{Keywords}

RSVP-TE, OPNET simulator

\section{INTRODUCTION}

Since the introduction of the world wide web, there has been a tremendous growth of the internet from just been a network of moderate proportional use, which was basically used by the academic community as well as for research and has now become a very large public data network, which plays a major role in the lives of people due to the large range of services and applications it offers and delivers. The high increase in the number of internet users made services such as telephone and television to reach their customers via the internet and this has been forcing Internet Service Providers (ISPs) to improve their quality of service. With this increase as well as the advances made in real-time applications (voice and video), the traditional routers have the challenges of providing the required high bandwidth, fast routing as well as quality of service support. Due to the challenges of traditional routers to provide these requirements especially for voice and video, methods such as the use of Multi-Protocol Label Switching (MPLS) and so on are now used. Multi-Protocol Label Switching (MPLS) is a fast growing technology, which plays a vital role in providing quality of service $(\mathrm{QoS})$ and traffic engineering. It uses information contained in the labels, which are attached to Internet Protocol (IP) packets to improve the fast forwarding of these packets. MPLS provides scalability as well as congestion control in order to overcome limitations such as high packet loss and excessive delays in the network.

Various researches [1-3] have been carried out on the comparison of IP and MPLS networks.

\section{BACKGROUND}

\subsection{Traditional IP Routing}

In the traditional IP routing, routing tables are built by every router in the network by the use of different routing protocols such as OSPF (Open Shortest Path First), RIP (Routing Information Protocol), IS-IS (Intermediate System-toIntermediate System) or BGP (Border Gateway Protocol). Every router in the network has to individually make routing decisions for each incoming IP packet after the routing tables are built and this is time consuming. A router checks its routing table at the arrival of a packet to verify the next hop for the packet based on the destination address of the packet given in the IP header of the packet.

\subsection{MPLS}

MPLS (Multi-protocol Label Switching) is an advancing technology, which is mainly responsible for high performance packet control and mechanism [4]. It does this by the information contained in the labels attached to the IP packets to forward such packets through a network. It merges the strength of layer 2 switching and layer 3 routing to form an IP network with a high level of performance. MPLS has evolved into a vital technology which efficiently operates and manages IP networks due to its superior characteristics [5]. The purpose of MPLS is to guarantee speed, traffic engineering, Quality of Service (QoS) and scalability of the network and is also useful for VPNs (Virtual Private Networks). MPLS is not a substitute for IP, but it extends the IP architecture by adding new functions to it. The MPLS domain has two major kinds of switches namely; the MPLS edge switches, which basically consist of the LERs (Label Edge routers) and the MPLS core switches, which basically consist of the LSRs (Label Switch Routers). When a packet enters into an MPLS domain, a label is attached to the packet. A label has no internal structure and is a short and fixed unit. This MPLS label is between the Data link layer and the Network layer and the packets are forwarded based on the MPLS labels.

\subsection{Traffic Engineering in MPLS Networks}

Traffic Engineering (TE) is a mechanism put in place to control the flow of traffic in networks and it provides the performance optimization of the network resources. The main characteristics of TE are fault-tolerance, optimum resource utilisation and resource reservation [6]. The basic objective of the consideration of TE is to improve quality of service of 
some applications and use the available network resources efficiently. There are some important factors, which are needed for TE. These factors are; Path Selection, Traffic Management, Direction of Traffic along Computed Paths and Distribution of Topology Information.

The LSPs in the MPLS network are established and the labels are distributed on each of the hops along the LSPs before packets could be forwarded. The LSPs can be established either by explicitly routed LSP or control driven LSP. Control driven LSPs can also be referred to as hop-by-hop LSP and are set by the use of LDP protocol. Explicitly routed LSPs can also be referred to as constraint based LSPS (CR-LSPs), which are specified in the setup message. At each hop, a label request is sent to the next hop along the LSP [7].

There are basically two protocols used to set CR-LSPs in MPLS. These protocols are; Resource Reservation Protocol (RSVP) and Constraint based routed LDP (CR-LDP).

\section{METHODOLOGY}

OPNET simulator was used to compare the three routing protocols. OPNET is a real-time simulator designed mainly for the design and analysis of network models [8]. The network topology used to carry out this research consists of 54 routers. Figure 1 below shows the IP network for this research and Figure 2 below shows the MPLS network for this research. The MPLS RSVP-TE network is the same as the MPLS network for this research except that the path R1 to R13 from the West_Router to the East_Router was reserved for the voice traffic.

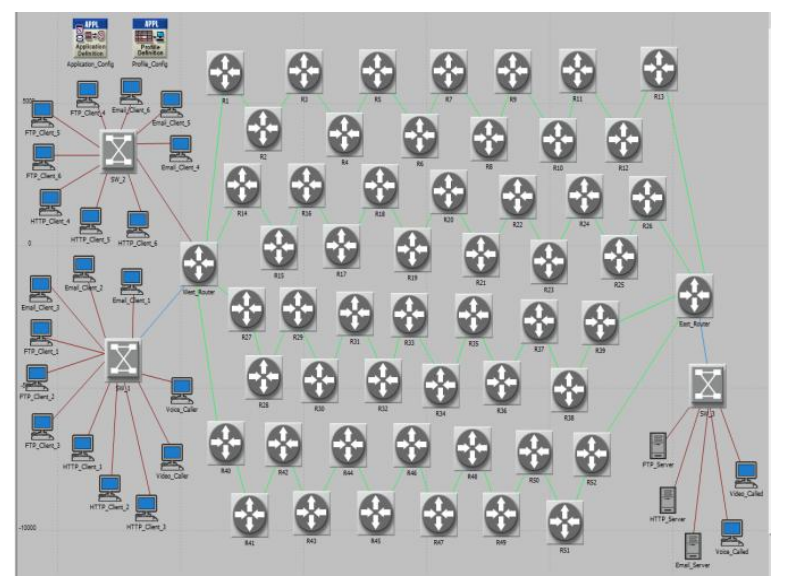

Figure 1: The IP Network

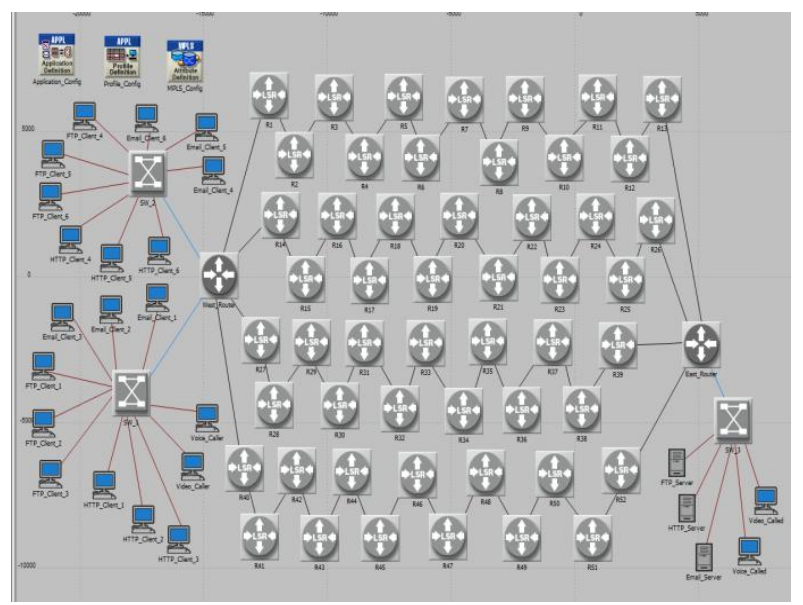

Figure 2: The MPLS Network

\section{SIMULATION RESULTS AND ANALYSIS}

\subsection{The Throughput}

From Figure 2 below, it can be seen that the throughput for the traditional IP network increased rapidly throughout the simulation period and was $19,051,127 \mathrm{bits} / \mathrm{sec}$ at the end of the simulation period. It can also be seen that the throughput for the MPLS network increased rapidly throughout the simulation period and was $18,110,513 \mathrm{bits} / \mathrm{sec}$ at the end of the simulation. Also, the throughput for the MPLS RSVP-TE network increased rapidly throughout the simulation period and was $16,752,371 \mathrm{bits} / \mathrm{sec}$ at the end of the simulation.

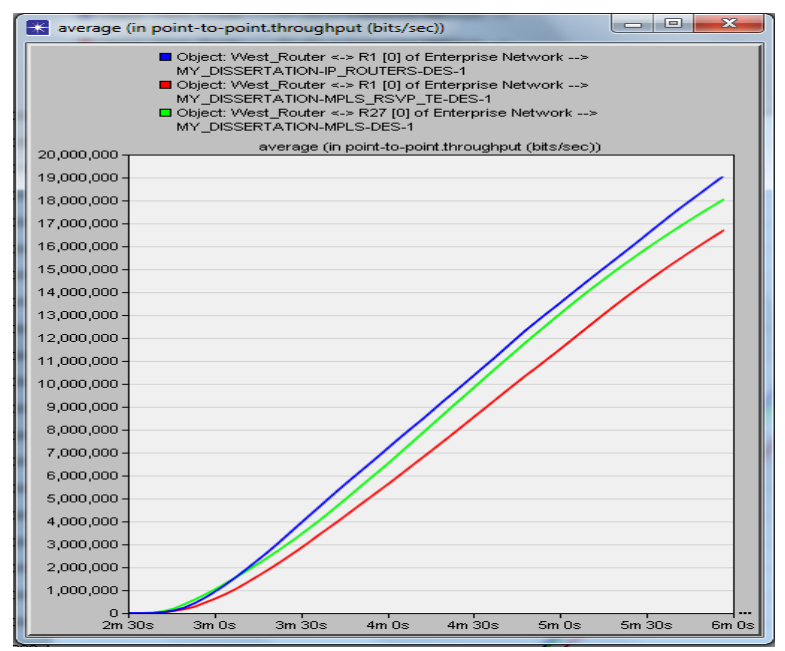

Figure 2: The Throughput

\subsection{The Utilisation}

From Figure 2 below, it can be seen that the throughput for the traditional IP network increased rapidly throughout the simulation period and was $19,051,127 \mathrm{bits} / \mathrm{sec}$ at the end of the simulation period. It can also be seen that the throughput for the MPLS network increased rapidly throughout the simulation period and was $18,110,513 \mathrm{bits} / \mathrm{sec}$ at the end of the simulation. Also, the throughput for the MPLS RSVP-TE network increased rapidly throughout the simulation period and was $16,752,371 \mathrm{bits} / \mathrm{sec}$ at the end of the simulation.

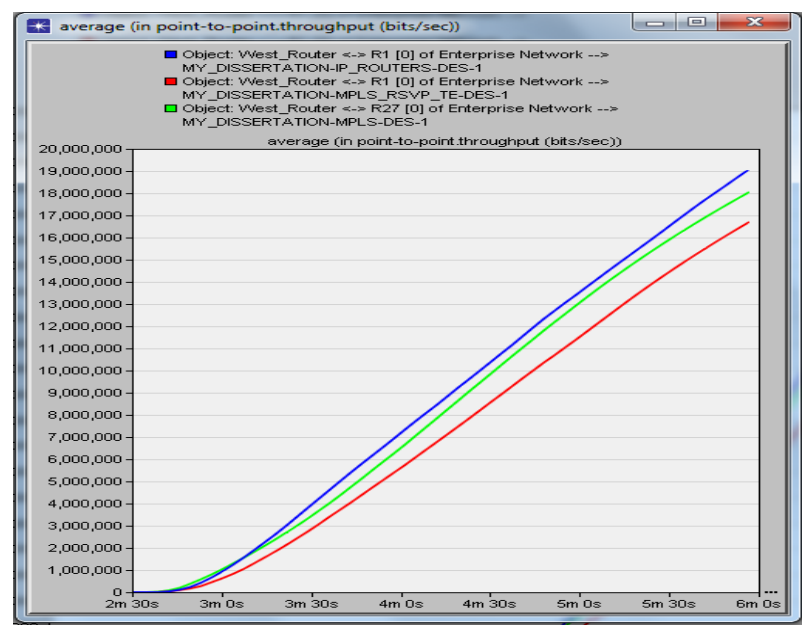

Figure 2: The Throughput 


\subsection{The Voice Jitter}

From Figure 4 below, it can be seen that the voice jitter for the traditional IP network was 0sec until the 3rd minute of simulation when it started to increase rapidly. At the end of the simulation, the voice jitter was $0.00052 \mathrm{sec}$. The voice jitter for the MPLS network was also 0sec until the 3rd minute of the simulation when it started to rapidly increase until it was $0.00032 \mathrm{sec}$ at the end of the simulation. The voice jitter for the MPLS RSVP-TE network was 0sec until the 4th minute when it gradually increased until it was $0.00006 \mathrm{sec}$ at the end of the simulation.

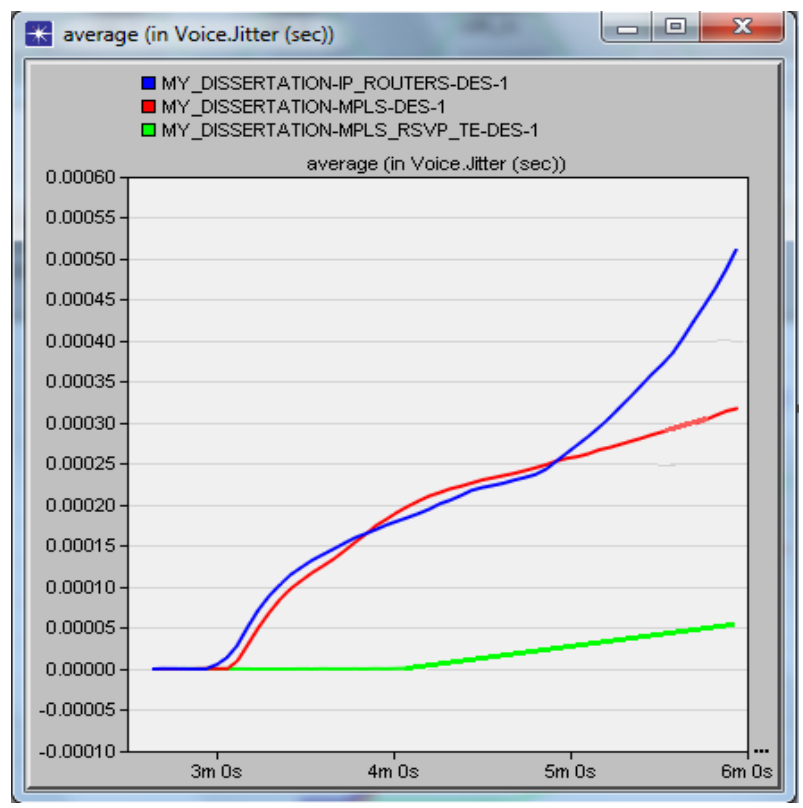

Figure 4: The Voice Jitter

\section{CONCLUSIONS}

After critically analysing the results, it can be said that the IP network has the highest throughput (higher than both the MPLS and MPLS RSVP-TE networks) and the MPLS network has a higher throughput than the MPLS RSVP-TE network. The throughput for the MPLS network is $95.06 \%$ of that of the IP network and that for the MPLS RSVP-TE network is $87.93 \%$ of that of the IP network.

It can also be said that the MPLS RSVP-TE network has the highest utilization (higher than both the MPLS and MPLS RSVP-TE networks) and the MPLS network has a higher utilization than the IP network. The utilization of the MPLS RSVP-TE network is about nineteen times that of the IP network while the utilization of the MPLS network is about seventeen times that of the IP network.
Furthermore, it can be said that the voice jitter in the IP network is approximately nine times that of the MPLS RSVPTE network and $0.0002 \mathrm{sec}$ higher than that of the MPLS network. The voice jitter in the MPLS network is approximately five times that of the MPLS RSVP-TE network. The MPLS RSVP-TE network has the least voice jitter due to the reserved path for the voice traffic in this network.

Therefore, it can be concluded that the MPLS network performs better than both the IP and MPLS RSVP-TE networks in terms of utilization while the MPLS RSVP-TE network has the best performance for voice traffic due to the reserved path and the MPLS network has a better performance for voice traffic than the IP network.

\section{ACKNOWLEDGMENTS}

All thanks to our parents and all those who supported us throughout the period of this research.

\section{REFERENCES}

[1] M.A. Rahman et al. "Performance Analysis of MPLS Protocols over conventional Network," Microwave Conf., China-Japan Joint, Shanghai, Sept. 2008, pp. 763766

[2] J. Barakovic et al. "Multimedia Traffic Analysis of MPLS and non-MPLS Network," Multimedia Signal Processing and Communications, 48th Int. Symp. ELMAR-2006, Zadar, Jun. 2006, pp. 285-288.

[3] R.S. Naoum and M. Maswady, "Performance evaluation for VOIP over IP and MPLS," World of Computer Science and Information Technology Journal (WCSIT), vol. 2, pp. 110-114, 2012.

[4] N. F. Mir and A. Chien, "Simulation of Voice over MPLS communications Networks," IEEE ICSS conf., CA, 2002, pp. 389-393.

[5] L. He and P. Botham, "Pure MPLS Technology," in Proc. 3rd International Conf. Availability, Reliability and Security, Barcelona, 2008, pp. 253-259.

[6] X. Xiao et al. "Traffic Engineering with MPLS in the Internet" Global Center Inc. and Michigan State University, USA, vol. 14, pp. 28-33, Mar. 2000.

[7] A. Ghanwani et al. "Traffic Engineering Standards in IP Networks Using MPLS" IEEE Communication Mag. Dec. 1999.

[8] H. M. Asif and M. G. Kaosar, "Performance Comparison of IP, ATM and MPLS Based Network Cores Using OPNET," in 1st IEEE Int. Conf. Industrial and Information Systems, Sri Lanka, Aug. 2006. 